\title{
The epistemology of the precautionary principle: two puzzles resolved
}

\author{
Asbjørn Steglich-Petersen \\ Aarhus University \\ filasp@cas.au.dk \\ Forthcoming in Erkenntnis
}

\begin{abstract}
In a recent paper in this journal (forthcoming), Carter and Peterson raise two distinctly epistemological puzzles that arise for anyone aspiring to defend the precautionary principle. The first puzzle trades on an application of epistemic contextualism to the precautionary principle; the second puzzle concerns the compatibility of the precautionary principle with the de minimis rule. I argue that neither puzzle should worry defenders of the precautionary principle. The first puzzle can be shown to be an instance of the familiar but conceptually harmless challenge of adjudicating between relevant interests to reach assessments of threats when applying the precautionary principle. The second puzzle can be shown to rely on a subtle but crucial misrepresentation of the relevant probabilities at play when applying the precautionary principle.
\end{abstract}

\section{Introduction}

The so-called precautionary principle is an increasingly influential decision rule or approach in environmental and healthcare decision-making. Although many different formulations of the principle are at play in various legal and political contexts, the basic idea motivating the principle is that if a potential effect of some activity is sufficiently detrimental to the environment or human health, we should take regulatory action against that activity even if there is significant epistemic or scientific uncertainty about the connection between the activity and the potential damage.

In a recent paper in this journal (forthcoming), Carter and Peterson (henceforth 'C\&P') raise two distinctly epistemological puzzles that arise for anyone aspiring to defend the precautionary principle. The first puzzle trades on an application of epistemic contextualism to the precautionary principle; the second puzzle concerns the compatibility of the precautionary principle with the de minimis rule.

In this note, I argue that neither puzzle should worry defenders of the precautionary principle. In §2 I show that the first puzzle is an instance of the familiar but conceptually harmless challenge of adjudicating between relevant interests to reach assessments of threats when applying the precautionary principle. In $\S 3$ I show that the second puzzle relies on a subtle but crucial misrepresentation of the relevant probabilities at play when applying the precautionary principle. 


\section{The first puzzle}

An obvious question raised by the precautionary principle is why threats of especially detrimental damage should be treated differently than threats of less severe damage, when it comes to the epistemic standards that must be met to warrant precautionary measures. What principled reasons could be given for lowering the epistemic standards to be met to warrant precautionary measures in cases of potentially severe consequences, compared to cases of less severe threats?

According to $\mathrm{C} \& \mathrm{P}$, an attractive explanation of this could appeal to a line of reasoning taken from contextualist or subject-sensitive invariantist (SSI) theories of knowledge. ${ }^{1}$ According to these theories, whether a person counts as knowing some proposition depends not only on the epistemic features of a situation, such as the person's evidence for the relevant proposition, but also on the practical stakes at play in the situation. To take the example provided by C\&P, suppose my evidence that the bank is open this Saturday consists in my having read a sign that says so. Defenders of contextualism and SSI allow that this evidence could be sufficient for knowing in a context in which little or nothing depends on whether the bank is open, while the very same evidence might fail to provide knowledge that the bank is open in a context in which someone's life or something similarly important depends on the bank being open. This is in opposition to 'strict invariantists', who hold that the practical stakes of a situation do not matter to whether someone counts as knowing. A significant part of the motivation for contextualism and SSI stems from the intuitive plausibility of standards of knowledge depending on what's at stake in cases such as the above.

C\&P observe that the same kinds of considerations that motivate contextualism and SSI to take epistemic standards to depend on practical stakes, might lead a defender of the precautionary principle to reason along broadly similar lines: the more severe the anticipated damage, the less certain we must be that some activity threatens that damage in order to be warranted in acting to regulate the activity. This would provide the defender of the precautionary principle the sought for principled explanation of why threats of especially severe damage should be treated differently than threats of less severe damages, when it comes to the epistemic standards that must be met to warrant regulation, since the explanation would be grounded in general epistemological considerations.

According to $\mathrm{C} \& \mathrm{P}$, however, this explanation faces a seemingly intractable challenge, which is not faced in the same way by contextualism and SSI in epistemology. The problem is

\footnotetext{
${ }^{1}$ For a contextualist approach, see e.g. DeRose (1992); for a recent critique, see Steglich-Petersen (forthcoming); for subject-sensitive invariantist approaches, see e.g. Hawthorne (2004) and Stanley (2005).
} 
that of determining whose interests are relevant, when determining the stakes, or the severity of some threat, in a situation where it might be relevant to apply the precautionary principle. Such situations are often characterized by conflicting interests of the different stakeholders. For example, a construction company wishing to build a road in Alaska will have different interests, than an environmental regulatory body fearing that the road will cause the extinction of a salmon species. These different interests will make the stakeholders evaluate the severity of the anticipated damage differently. According to $\mathrm{C} \& \mathrm{P}$, this means that a contextualist approach to the precautionary principle [...] could be plausible as a decision rule only if supplemented with some additional favouring rule, a rule which adjudicates whose interests determine the severity of the damage in question, a severity that (for the contextualist) is what fixes the relevant epistemic standard that must be satisfied (p. 7).

C\&P puts the issue in the form of a dilemma: On the one hand, if a defender of the precautionary principle goes the invariantist route and denies that the epistemic standards that must be met when applying the precautionary principle depend on the severity of the anticipated damages, she lacks any principled way to explain why threats of (say) massive irreversible catastrophe warrant special treatment, i.e. why we need to know less to justifiably restrict threatening activities (p.6). On the other hand, if a defender of the precautionary principle goes the contextualist/SSI route, she will face the difficult question of deciding whose interests are relevant to assessing the anticipated damage.

The comparison between the precautionary principle and contextualist/SSI approaches in epistemology is promising, and should be explored further. It should be clear, however, that the defender of the precautionary principle shouldn't be particularly perturbed by the dilemma outlined by C\&P. In particular, she shouldn't be worried by the problem they raise for the contextualist/SSI approach out of the dilemma.

To see this, note first that quite independently of any epistemic considerations, any use of the precautionary principle as a decision rule will involve adjudicating between competing interests, since assessing the costs of potential threats and precautionary measures, as well as the potential value of what's to be saved by those measures, will be hostage to the often competing interests involved in the decision scenario. In fact, this is a well-known difficulty when applying the precautionary principle in policy making. Rarely, however, is this difficulty seen as an argument against the validity or usefulness of the precautionary principle, since any decision rule which relies on assessments of costs and values, will be subject to this kind of prior adjudication of interests, especially when applied in policy making. Nor is it an argument 
for adopting some additional favoring rule that tells us how to adjudicate between the relevant interests in policy making. Such adjudication will typically be done through the normal political processes, sometimes democratic, which are designed to facilitate adjudication of that exact kind. Making the epistemic standards involved in applying the precautionary principle depend on the relevant interests does not raise any new additional difficulties, since it is the very same adjudication that is involved when fixing the relevant costs and values, that would also affect whether the epistemic standards are met. In other words, once the normal adjudication of interests involved when applying the precautionary principle in policy making has taken place, deciding whether the epistemic standards are met would not require any new and additional adjudication of interests. One may think, of course, that the inevitable and wellknown need for interest-adjudication when applying the precautionary principle is a more serious problem than indicated above. But in that case, this would not be a problem arising from the contextualist approach to the epistemic standards involved with the precautionary principle, and C\&P would therefore not have succeeded in pointing out a new puzzle for the precautionary principle. ${ }^{2}$ With this in place, we can move on to consider the second epistemological puzzle raised by $\mathrm{C} \& \mathrm{P}$.

\section{The second puzzle}

The point of departure for the second puzzle is the, according to C\&P, widely accepted claim that any reasonable formulation of the precautionary principle must be compatible with the $d e$ minimis principle, according to which sufficiently improbable risks, falling under some threshold of probability, should be ignored. ${ }^{3}$ Otherwise, even the most trivial activities raising the slightest possibility, however improbable, of some catastrophic threat, should be subject to the precautionary principle, which seems implausible.

The puzzle arises once we notice that the relevant probability when determining whether some risk is de minimis couldn't simply be the first-order probability of the undesirable outcome. We would also have to take into account a second-order probability,

\footnotetext{
${ }^{2}$ It may be objected that the whole idea of the precautionary principle is to mark a departure from standard decision procedures, such as cost-benefit analysis or standard risk-analysis, in not having to take into account and adjudicate between the interests of opposing stake-holders, and instead provide a more direct decision procedure in acting to protect the environment and human health. While I am sympathetic to this understanding of the precautionary principle, I will not pursue it further here, but only note that if this understanding is correct, and application of the precautionary principle does not involve adjudication of interests, a central presupposition of C\&P's puzzle from contextualism is false, thus dissolving the puzzle. I thank an an'onymous reviewer for bringing this to my attention.

${ }^{3}$ Among the authors listed as supporters of this claim by C\&P are Peterson (2002), Sandin (2004: 21), Clarke (2005), Steele (2006), Ashford (2007), and Zander (2010: 72).
} 
which tells us how reliable or certain the estimate of the first-order probability is. To use C\&P's own illustration, imagine that you estimate the probability that a person will get cancer from substance $X$ to be one in a million. This is in itself not sufficient for concluding that the risk is de minimis, because your assessment of the probability may be highly uncertain. This type of uncertainty can be rendered more precise by distinguishing between first-and secondorder probability. In our example, the first-order probability is the probability that substance $X$ will cause cancer. The second-order probability refers to the probability that the assessment of the first-order probability is correct (p. 9).

Clearly, both types of probability are relevant when deciding whether a potential risk calls for precautionary measures or is de minimis. But how can we take both types of probability into account when deciding this? Once again, C\&P claim that the defender of the precautionary principle meets a dilemma. The first horn of the dilemma arises if we try to combine the two probabilities into a single all-things-considered measure. C\&P claim that the most obvious way of doing so delivers the intuitively wrong results, and that an alternative way that delivers the right results would be ad hoc. I will return to this argument in a moment. The second horn of the dilemma arises if we refrain from combining the two probabilities into a single measure, and instead consider each type separately. The problem with this approach, according to $\mathrm{C} \& \mathrm{P}$, is that we would have to introduce threshold probabilities for de minimis for both probabilities, but as soon as we introduce two or more thresholds, we can always imagine cases in which small changes to one threshold lead to a 'too big' effect on the overall assessment (p. 11). I agree with C\&P that this approach is hopeless, and shall not dwell on it any further. But contrary to $\mathrm{C} \& \mathrm{P}$, the prospects of the first approach of combining the probabilities into a single measure seem bright. The problems C\&P see stem from a subtle but decisive misrepresentation of the precautionary principle.

$\mathrm{C} \& \mathrm{P}$ motivate the problem for the combining strategy by first considering a very simple procedure for combining the two kinds of probability into a single measure, namely by multiplying them. C\&P are of course well aware that any remotely plausible way of combining first- and second-order probabilities will be more complex than this, but they assume that the problem illustrated by the simple procedure generalizes to the more complex approaches. ${ }^{4}$ As $\mathrm{C} \& \mathrm{P}$ note, multiplying the two probabilities will not give the right result with respect to de minimis, because if we multiply a small number (the first-order probability) by another small number (the second-order probability), then the all-things-considered probability will be even

\footnotetext{
${ }^{4}$ For a recent discussion of how to aggregate first- and second probabilities, see e.g. Hansson (2008).
} 
smaller. [...] It then follows that the less certain we are that the second-order assessment [of the first-order probability] is correct, the smaller will the all-things-considered risk be [...]. This is clearly the wrong conclusion. Intuitively, it would make more sense to apply the precautionary principle if the first-order probability is highly uncertain, which is the opposite of what the multiplicative rule suggests (p. 10).

If this problem for the simple multiplication approach generalizes to more complex aggregating approaches, we thus face a tension between two opposing intuitions. If the secondorder probability is low, the relevant all-things-considered probability should be even lower, since it will then be less likely that the first-order probability is correct, thus making the precautionary principle less likely to be applicable. But contrary to this, C\&P claim, it seems that a low second-order probability is actually a reason in favor of applying the precautionary principle, and not declaring the risk de minimis.

It is worth pausing briefly at this stage to note that in citing a low second-order probability as a reason in favor of applying the precautionary principle, $\mathrm{C} \& \mathrm{P}$ seem to rely on the non-trivial assumption that a low second-order probability results in an increased overall risk. It should be clear, however, that a low second-order probability doesn't on its own have any such effect. Suppose that I live in Baltimore and read in the weather forecast that there is a 0.8 probability of thunderstorms, but subsequently come to doubt that I have read the forecast for Baltimore and not Chicago (perhaps I set my phone to sometimes report the one, sometimes the other), and assign it a 0.5 probability that I have read the right forecast. What does this relatively low second-order probability imply about my overall risk of encountering thunderstorms in Baltimore? Nothing, it seems. The second-order probability of 0.5 favors an overall risk higher than the first-order probability of 0.8 , as much as it favors an overall risk lower than 0.8 . The only way that the low second-order probability could increase the overall risk of thunderstorms in Baltimore is if we are biased towards risk in our probability assessments, making the background assumption one of danger, rather than safety. As I shall go on to show, this is not an unreasonable assumption in the context of applying the precautionary principle, but for now it should just be noted that C\&P's puzzle tacitly relies on it. ${ }^{5}$

Returning to the tension outlined above, $\mathrm{C} \& \mathrm{P}$ note that an easy solution would be to 'turn around' the probability, and instead of calculating the all-things-considered probability that some activity is dangerous, calculate the probability that the activity is safe. It would follow, then, that the all-things-considered probability of safety is lower than the first-order

\footnotetext{
${ }^{5} \mathrm{I}$ am grateful to an anonymous reviewer for raising this issue.
} 
probability of safety, and that a lower second-order probability results in a lower probability of safety, which is the correct result. But C\&P are quick to dismiss this solution as being ad hoc. Although we get the result we want, C\&P claim that we have no good explanation why this way of carrying out the calculation is correct, and other way incorrect. However, as I shall now argue, there is nothing ad hoc about this solution at all. In fact, this solution is much more faithful to the spirit of the precautionary principle, than C\&P suggest. To see this, it will be useful to consider C\&P's basic understanding of the precautionary principle.

According to $\mathrm{C} \& \mathrm{P}$, the precautionary principle recommends regulatory action when there is sufficiently strong epistemic ground for thinking that some activity threatens sufficiently severe damage, i.e. when the risk or probability of severe damage is sufficiently high $(\mathrm{C} \& \mathrm{P}, \S 2)$. On this construal, it is the proponent of precautionary regulation that must show that some target activity threatens damage with a sufficiently high probability, before the precautionary principle can be applied. But as is widely recognized in the literature, one of the core ideas behind the precautionary principle is actually a reversal of the burden of proof in contexts of uncertainty. As one of the most influential formulations, the 1998 Wingspread Consensus Statement of the Precautionary Principle, puts it:

When an activity raises threats of harm to human health or the environment, precautionary measures should be taken even if some cause and effect relationships are not fully established scientifically. In this context, the proponent of an activity, rather than the public, should bear the burden of proof. (My emphasis)

Another illustrative formulation can be found in the United Nations' so-called Earth Charter, which states that,

When knowledge is limited apply a precautionary approach [...]. Place the burden of proof on those who argue that a proposed activity will not cause significant harm, and make the responsible parties liable for environmental harm.

Yet another influential formulation, which is adopted by numerous environmental NGOs, and is presented by the Wikipedia article on the precautionary principle as a 'core' idea of the precautionary principle, states that,

Under the precautionary principle it is the responsibility of an activity proponent to establish that the proposed activity will not (or is very unlikely to) result in significant harm.

In other words, the precautionary principle should primarily be seen as mandating regulatory action whenever the activity proponent has failed to lift their burden of proof, i.e. when there is insufficient certainty about the safety of an activity, not (just) when there is sufficient certainty about the threat raised by an activity. ${ }^{6}$

\footnotetext{
${ }^{6}$ For an additional recent discussion of the precautionary principle supporting the reading of this principle as essentially involving a reversed burden of proof, see Ahteensuu (2013).
} 
Granted, there are other formulations of the precautionary principle that make this core idea of a shifted burden of proof less explicit, but often, such formulations, including the one found in the Rio Declaration, are consistent with it. Even if there is a minority of formulations, such as the one quoted by C\&P from a British Government White Paper on environmental management, which state that the precautionary principle applies in particular where there are good grounds for judging [...] that irreversible effects may follow if action is delayed, it would certainly not be ad hoc in the present context to adopt what seems to be the more common understanding of the precautionary principle, as mandating precautionary measures when there is uncertainty about the safety of an activity. ${ }^{7}$

If we adopt this decidedly non-ad hoc understanding, the puzzle of how to combine first- and second-order probabilities goes away, since the relevant all-things-considered probability will be the probability that some target activity is safe. This means that the lower the second-order probability, the lower the all-things-considered probability of the activity being safe. This is the right result, since the second-order probability measures the reliability of the first-order assessment, and a lower second-order probability should therefore correspond to a reduced probability of safety. A risk would then be deemed de minimis when the probability of the activity being safe is sufficiently high, or, which is to say the same, when the probability of harm is sufficiently low. And just as we wanted, a low second-order probability becomes a reason in favor of applying the precautionary principle, and not declaring the risk de minimis.

It should be noted that, just as C\&P's puzzle did, this solution relies on the non-trivial assumption that a low second-order probability results in an increased overall risk. As we saw, this can only be the case if we are biased towards risk in our probability assessments, making the background assumption one of danger, rather than safety. But it should be obvious that such a bias coheres well with the interpretation of the precautionary principle as imposing a burden of proof on activity proponents. Indeed, a bias towards risk seems part and parcel with activity proponents carrying the burden of proof, since this allows us to assume that an activity is unsafe unless the activity proponent can show it not to be.

\footnotetext{
${ }^{7}$ An alternative interpretation of the quoted passage from the British Government White Paper, which would make it consistent with the core idea of a shifted burden of proof and thereby also consistent with the above quoted formulations of the precautionary principle, would understand the requirement of 'good grounds [...] that irreversible effects may occur' as a sort of initial test prior to applying the precautionary principle, and thereby the shifted burden of proof. For example, we may have good grounds for judging that some activity entails the possibility ('may occur') of some irreversible effect, without yet having determined the probability of this, in which case the precautionary principle would apply to demand that those responsible for the activity establish that the harmful effects are sufficiently improbable for the activity to be allowed.
} 
Incidentally, this way of understanding the precautionary principle also strengthens the connection between the precautionary principle and the intuitions from contextualist and SSI approaches in epistemology that $\mathrm{C} \& \mathrm{P}$ trade on. In the course of discussing this connection, $\mathrm{C} \& \mathrm{P}$ rightly note that both the precautionary principle and contextualism/SSI make epistemic standards depend on what's at stake in the relevant situation. But as $\mathrm{C} \& \mathrm{P}$ are aware, on their construal of the precautionary principle, there is a crucial difference between these dependencies, which shows up in the respective correlations at play. In the case of contextualism/SSI, the correlation is positive: the more significant the practical stakes, the higher the epistemic standards one must meet to qualify as possessing knowledge. But on C\&P's understanding of the precautionary principle, the correlation found here is negative or inverse: the more severe the anticipated damage, the less certain we must be that some activity threatens that damage in order to be warranted regulating that activity. $\mathrm{C} \& \mathrm{P}$ does not comment further on this puzzling outcome of their comparison. If we instead adopt the construal of the precautionary principle recommended above, however, the correlation between epistemic standards and what's at stake becomes positive instead of negative, thus bringing it into line with the correlation exhibited by contextualism and SSI. On this understanding, the precautionary principle has it that the more severe the potential damage, the more certain we must be that some activity is safe in order to be warranted in not regulating that activity. This alignment of correlations can be taken as further circumstantial evidence that this is the correct way of understanding the precautionary principle. It also strengthens the case for further investigating the links between the precautionary principle and certain key claims of contextualist/SSI approaches in epistemology. But such an investigation must be left for another occasion.

\section{Conclusion}

In conclusion, neither of the puzzles raised by $\mathrm{C} \& \mathrm{P}$ should worry defenders of the precautionary principle. The first puzzle is just a special case of the familiar but conceptually harmless challenge of adjudicating between relevant interests to reach assessments of threats when applying the precautionary principle in policy-making. This well-known challenge should not be seen as a reason to search for some additional decision-theoretic favoring rule, but rather be seen as an inevitable part of the political process of reaching alignment between opposing interests. The second puzzle can be shown to rely on a subtle but crucial misrepresentation of what can plausibly be seen as one of the core ideas behind the precautionary principle, namely 
the shifted burden of proof. This affects how we should see the relevant probabilities at play when applying the precautionary principle, thus yielding the right results when combining firstand second-order probabilities, and the right de minimis judgments.

\section{References}

Ahteensuu M (2013). The Precautionary Principle and the Justifiability of Three Imperatives. Homo Oeconomicus, 30, 17-36.

Ashford, N. A. (2007). The legacy of the precautionary principle in US law: The rise of cost-benefit analysis and risk assessment as undermining factors in health, safety and environmental protection." Implementing the precautionary principle. Approaches from the Nordic Countries, EU and USA. London: Earthscan.

Carter, J.A., \& Peterson, M. (forthcoming). On the epistemology of the precautionary principle. Erkenntnis.

Clarke, S. (2005). Future technologies, dystopic futures and the precautionary principle. Ethics and Information technology, 7, 121-126.

DeRose, K. (1992). Contextualism and knowledge attributions. Philosophy and Phenomenological Research, 52, 913-929.

Hansson, S.O. (2008). Do We Need Second-Order Probabilities? Dialectica, 62, 525533.

Hawthorne, J. (2004). Knowledge and lotteries. Oxford: Clarendon.

HM Government (1990). White Paper: This Common Inheritance: Britain’s Environmental Strategy.

Peterson, M. (2002). What is a de minimis risk? Risk Management, 47-55.

Sandin, P. (2004). The precautionary principle and the concept of precaution. Environmental Values, 13, 461-475.

Steele, K. (2006). The precautionary principle: a new approach to public decisionmaking? Law, Probability and Risk, 5, 19-31.

Steglich-Petersen, A. (forthcoming). Knowing the answer to a loaded question. Theoria.

UN General Assembly (1992). Rio declaration on environment and development. Agenda, 21.

United Nations. The Earth Charter. http://www .earthcharterinaction.org/content/pages/Read-the-Charter.html

Stanley, J. (2005). Knowledge and practical interests. Oxford University Press. 
Wikipedia. Precautionary Principle.

http://en.wikipedia.org/wiki/Precautionary_principle

Wingspread Statement on the Precautionary Principle (1998).

http://www.sehn.org/wing.html.

Zander, J. (2010). The application of the precautionary principle in practice:

comparative dimensions. Cambridge University Press. 